\title{
A Rare Case of Mixed Tumor of Right Nasal Sill Diagnosed Histopathologically more Frequently than Expected
}

\author{
Tan Shi Nee N,2,3*, Rajarajeswari Arunathan $^{2}$ and Mohd Shakir Bathusha ${ }^{4}$ \\ ${ }^{1}$ Department of Otorhinolaryngology-Head and Neck Surgery, Hospital Tawau, Malaysia \\ ${ }^{2}$ Department of Otorhinolaryngology-Head and Neck Surgery, Hospital Lahad Datu, Malaysia \\ ${ }^{3}$ Department of Otorhinolaryngology-Head and Neck Surgery, KPJ University College, Malaysia \\ ${ }^{4}$ Department of Plastic, Reconstructive and Burns, Hospital Sultanah Nur Zahirah, Malaysia
}

*Corresponding author: Tan Shi Nee, Department of Otorhinolaryngology-Head and Neck Surgery, Hospital Lahad Datu, Sabah, Malaysia.

Received Date: April 28, 2020

Published Date: May 11, 2020

\begin{abstract}
Mixed tumor (MT) is an unusual, benign skin appendageal tumor, with an eccrine and apocrine origin, resembling mixed tumors of the salivary glands. Although it usually involves the head and neck region, the nasal region is a rare location. It develops as a slow-growing, small cutaneous mass. We report a rare case of mixed tumor in a 35-year-old female, who presented with a firm, asymptomatic mass in the right nasal sill, slowly growing for approximately 5 years. A provisional clinical diagnosis was made by fine needle aspiration cytology evaluation. The final diagnosis is confirmed by histopathological examination. This case highlights the importance of fine needle aspiration cytology in providing the essential clue to uncover the diagnosis and orient the surgical management of the patient. The excision and repair techniques require more skill and time, considering the location of the lesion.
\end{abstract}

Keywords: Chondroid syringoma; Mixed tumor; Pleomorphic adenoma; Fine needle aspiration cytology; Skin adnexal tumors

\section{Introduction}

Mixed tumor (MT) is an unusual, rare benign skin appendageal tumor with an eccrine and apocrine origin, resembling mixed tumors of the salivary glands. It was first described by Hirsch and Helwig which was previously named as Chondroid Syringoma (CS) due to the presence of sweat gland elements in the cartilaginous stroma [1]. The incidence of the mixed tumor is low (about $0.01 \%$ reported) [2, 3]. It usually involves the head and neck region developing as a slow-growing, small cutaneous mass. Because of its non-specific presentation and rare incidence, the diagnosis of MT is usually made by the histopathologic report [3]. Herein, we report a case with a histologically proven mixed tumor of the nasal sill. The review of the literature on this topic (mixed tumor located in the nasal region) is extremely limited in this manuscript.

\section{Case Presentation}

A 35-year-old female with unremarkable medical history presented with a mass located in the right nasal still that had been developing for 5 years. The swelling started as small and slowly progressing nodule (Figure 1). The swelling was not painful and no other tumors were noted elsewhere. There was no history of trauma or spontaneous bleeding. No history was suggestive of systemic illnesses. Local examination revealed a firm to hard mass measuring about $2 \times 2.5 \mathrm{~cm}$ situated over the right nasal sill. It was mobile, non-fluctuant, non-tender fixed to the skin, and freely mobile over the underlying structure. No regional lymphadenopathy was observed. A provisional diagnosis of the right nasal sill sebaceous cyst was made. The fine needle aspiration 
cytology performed before operation suggested a benign lesion where there are clusters of spindle cells that have oval nuclei with an abundance of well-defined cytoplasm and are embedded in a chondromyxoid substance.

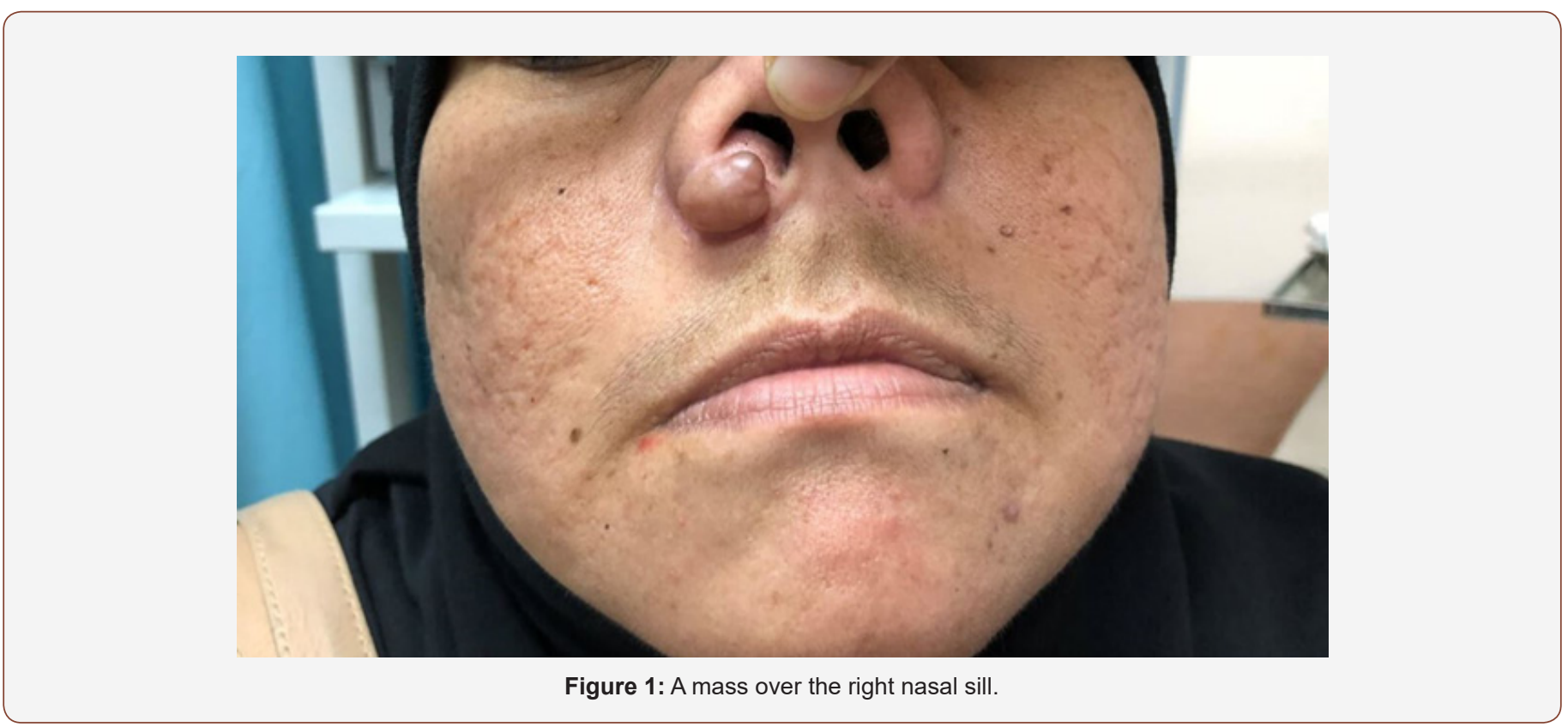

Our patient underwent an excisional biopsy of the right nasal sill mass with histopathological examination. Grossly, the excised tumor mass with attached elliptical superjacent skin measured $4 \times 2.5 \mathrm{~cm}$. Its external surface was smooth (Figure 2). Microscopically, the section showed an intradermal, thin encapsulated circumscribed tumor tissue composed of a mixture of an epithelial and mesenchymal component which suggestive of more eccrine origin. The epithelial components consist of ductal and myoepithelium. The ductal epithelium lined the ducts and composed of rows of cuboidal cells. Post-operative, the procedure was uneventful (Figure 3). On 2nd and 6th month follow-up in the outpatient clinic, the patient was well, with excellent wound healing and no complications or local recurrence.

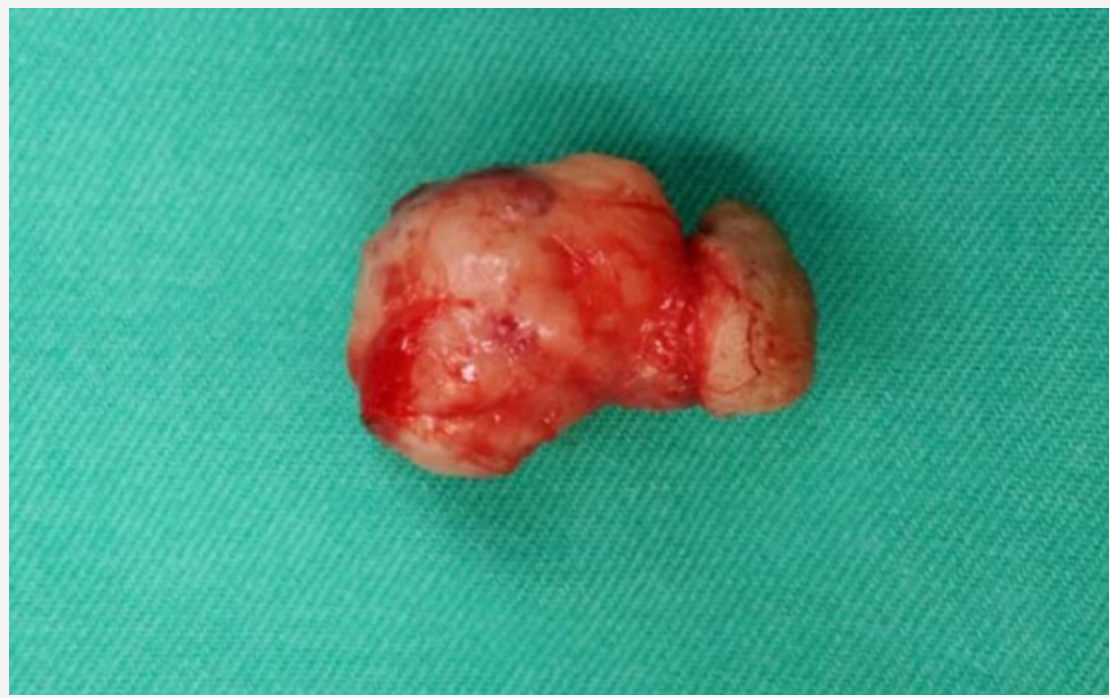

Figure 2: A picture showing the excised tumor mass with an attached elliptical piece of skin.

\section{Discussion}

Mixed tumor (MT) is a rare tumor which is also known as chondroid syringoma. Hirsch and Helwig first introduced the term CS [1]. It is later introduced bu Virchow and Minssen [4] of the new term, mixed tumor of the skin because it represents benign tumor with the cutaneous (epithelial) part and mesenchymal in origin [5].

Five histological criteria were proposed to diagnose chondroid syringoma for these tumors, by Hirsch and Helwig in 1961 [1]:

1) Interconnected tubuloalveolar structures which were lined with two or more rows of cuboidal cells. 
2) Nests of cuboidal or polygonal cells.

3) Ductal structures which were composed of one or two rows of cuboidal cells.
4) Occasional keratinous cysts.

5) A matrix of varying composition.

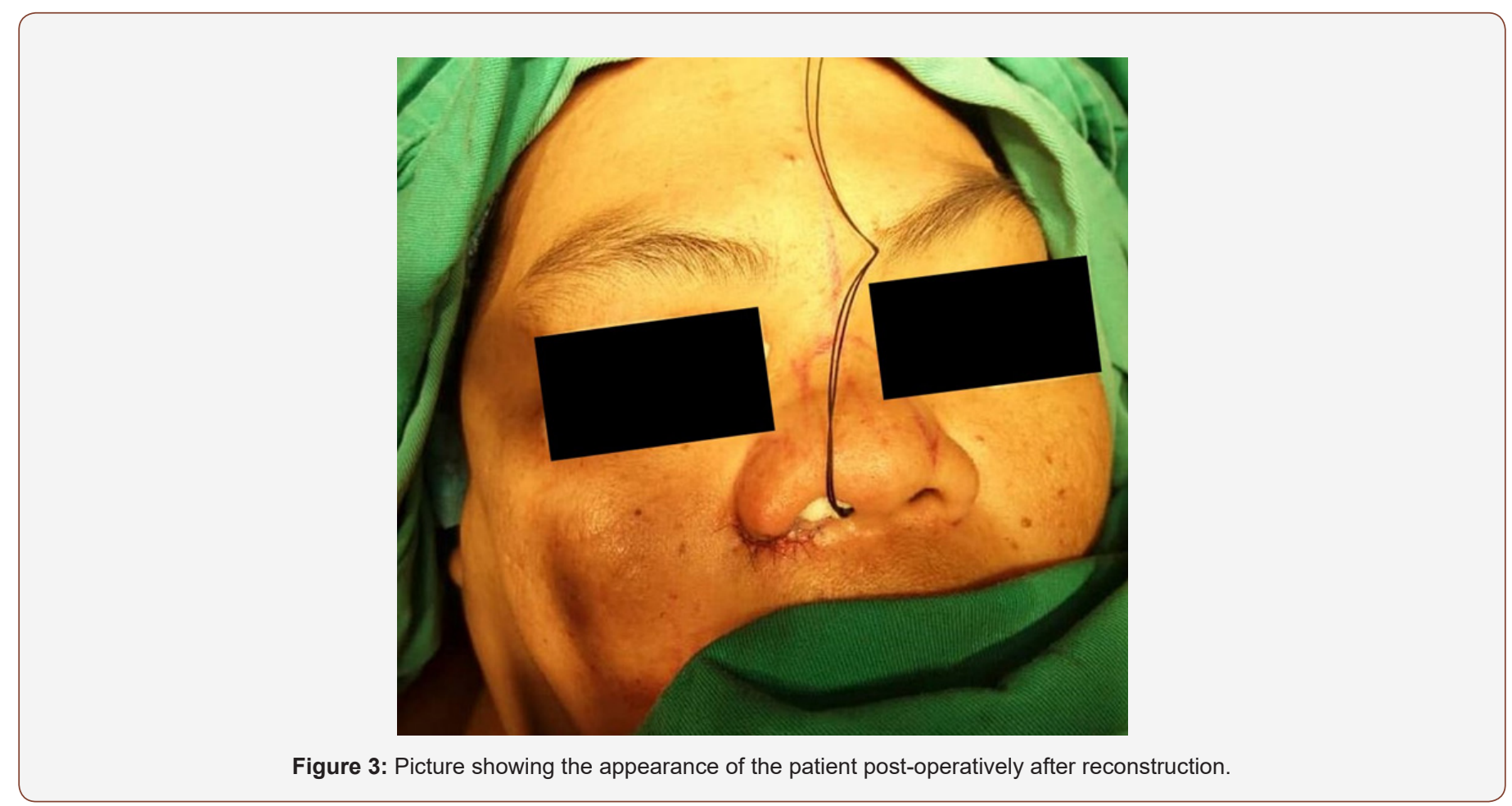

CS may have all the five histological criteria or they may have only one characteristic.

As a rare entity, the mixed tumor may not typically be part of the differential diagnosis of a slow-growing subcutaneous mass in this region. From a histopathological perspective, its differentiation towards various skin adnexal structures which include hair follicle, apocrine, sebaceous gland, and hair matrix may not always be apparent and it may be confused with other more common skin lesions of epidermal or mesenchymal appendages such as a dermoid cyst, sebaceous cyst, epidermal cyst and neurofibroma, lipoma, etc [6].

Fine needle aspiration cytology (FNAC) is a good guide as initial planning of diagnostic purpose and it may be useful to determine possible pathology before excision [7]. However, most of the time, histopathological examination of the surgical specimen is key to establishing a correct and complete diagnosis of CS. Besides that, mixed tumor or known as chondroid syringoma share similarities with pleomorphic adenoma. The differences between both are, pleomorphic adenoma is a mixed tumor that arises from salivary glands and chondroid syringoma are mixed tumor arise from the sweat glands $[6,8]$.

Most of the time, the usual clinical presentation is that of an asymptomatic slow-growing mass in the region of the head and neck. In our case, the tumor is located in the right nasal sill, and our patient presented to the clinic because of aesthetical concerns. Other consequences of the slowly growing mass over the nasal site are nasal obstruction, aesthetically disfigured, pain, and risk of infection. Presently, there is a multitude of treatment options for chondroid syringoma such as carbon dioxide laser (CO2 laser), electrodesiccation, and vaporization with argon or CO2 laser [8]. However, complete surgical excision with negative margins is the preferred treatment choice $[3,6]$ to prevent a high rate of recurrence.

Such treatment options were preferred surgical excision because they are safe, without adverse scarring, textural changes, an aesthetically and functionally reliable method that can eradicate the tumor. The tumor clearance rate increased as complete surgical excision is possible. Hornick and Fletcher reported a series of 33 cases of benign low-grade cytology where they showed a total of 6 patients (18\%) that developed local recurrence, however, there were no clinical or histologic features reported with the recurrence [9]. A study done by Hornick and Fletcher was done to characterize this tumor further and they showed that although the majority are a morphologically benign myoepithelial tumor of soft tissue or mixed tumor, there is still a risk for local recurrence. In our case, the location of the tumor in the right nasal sill was a challenge, as we were not only aiming to perform a complete excision with negative margins but also careful to preserve the aesthetics and functionality of the affected structures. It is also important to closely follow-up the patient because of the risk of local recurrence [10].

\section{Conclusion}

Although it is known that CS usually occurs in the head and 
neck region, surgeons seldomly consider CS in their differential diagnosis as there are more common entities typical to this location. In middle-aged patients, CS should always be kept in mind when presenting as an asymptomatic, soft tissue mass, as this may easily be treated by simple excision.

\section{Source of Support: Nil}

Conflict of Interest: No potential conflict of interest relevant to this article was reported.

\section{References}

1. Hirsch P, Helwig EB (1961) Chondroid syringoma. Mixed tumor of the skin, salivary gland type. Arch Dermatol 84: 835-847.

2. Kerimoglu U, Aydingoz U, Ozkaya O, Aksu AE, Ergen FB (2006) MRI of a benign chondroid syringoma. Br J Radiol 79(944): e59-61.

3. Yun YM, Shin S, Kyung H, Song SH, Kang N (2016) Recurrent Chondroid Syringoma of the Alar Rim. Arch Craniofac Surg 17(1): 35-38.

4. Barman DC, Bhowmik A (2016) An unusual presentation of chondroid syringoma. Indian J Pathol Microbiol 59: 362-364.
5. R Yavuzer, Y Basterzi, A Sari, Ferda Bir, Cem Sezer, et al. (2003) Chondroid syringoma: a diagnosis more frequent than expected. Dermatol Surg 29(2): 179-181.

6. Askari K, Ghorbani G, Yousefi N, Saadat SM, Saadat SN, et al. (2014) Chondroid syringoma of the forearm: a case report of a rare localization. Indian J Dermatol 59(5): 507-509.

7. Young Mook Yun, Seungho Shin, Hyunwoo Kyung, Seung Han Song, Nakheon Kang (2016) Recurrent Chondroid Syringoma of the Alar Rim. Arch Craniofac Surg 17(1): 35-38.

8. Syed MA, Paudel U, Rajbhandari A, Pokhrel DB, Adhikari RC, et al. (2019) Fine needle aspiration cytology as a preliminary diagnostic tool in chondroid syringoma: a case report and review. Clin Cosmet Investig Dermatol 5(12): 209-218.

9. Solanki LS, Dhingra M, Bhalla M, Thami GP, Punia RP, et al. (2011) Chondroid syringoma: report of two cases in young patients. Dermatol Online J 17(12): 1-7.

10. Hornick JL, Fletcher CD (2003) Myoepithelial tumors of soft tissue: a clinicopathologic and immunohistochemical study of 101 cases with evaluation of prognostic parameters. Am J Surg Pathol 27: 1183-1196. 